ina morsitans morsitans. Fournal of Protozoology, 26, 557-563.

Hoare, C. A. (1972). The trypanosomes of mammals: a zoological monograph. Oxford: Blackwell Scientiffic Publications.

Jenni, L., Molyneux, D. H., Livesey, J. L. \& Galun, R. (1980). Feeding behaviour of tsetse flies infected with salivarian trypanosomes. Nature, 283, 383-385.

Livesey, J. L., Molyneux, D. H. \& Jenni, L. (1980). Mechanoreceptor-trypanosome interaction in the labrum of Glossina: fluid mechanics. Acta Tropica, 37, 151-161.

Molyneux, D. H. (1980). Host-trypanosome interactions in Glossina. Insect Science and its
Applications, I, pp. 39-46.

Molyneux, D. H., Lavin, D. \& Elce, B. (1979). A possible relationship between salivarian trypanosomes and Glossina labrum mechanoreceptors. Annals of Tropical Medicine and Parasitology, 73, 287-290.

Rice, M. J., Galun, R. \& Margalit, (1973). Mouthpart sensilla of the tsetse fly and their function. III Labrocibarial sensilla. Annals of Tropical Medicine and Parasitology, 67, 109-116.

Vickerman, K. (1973). The mode of attachment of Trypanosoma vivax in the proboscis of the tsetse fly Glossina fuscipes: an ultrastructural study of the epimastigote stage of the trypanosome. Fournal of Protozoology, 20, 394-404.

\title{
Characterization of antigens from erythrocytic stages of Plasmodium falciparum reacting with human immune sera*
}

\author{
Luc H. Perrin, Renu Dayal and H. Rieder \\ WHO Immunology Research and Training Centre and Department of Medicine, Hospital Cantonal, \\ 1211 Geneva 4, Switzerland
}

Epidemiological studies and the experimental data of MCGREGOR \& WILlIAMS (1978) in the Gambia have shown that, in areas endemic for malaria, humans acquire a partial immunity to malaria infection after repeated infections, as rcflected, for example, by a decrease in the number of individuals with patent parasitaemia with age. There is evidence that humoral factors play a major role in this process. It has been shown, for example, that passive transfer of immunoglobulins from adults living in hyperendemic areas of West Africa can suppress the parasitaemia of children suffering from Plasmodium falciparum and $P$. malariae infections (COHEN et al., 1961). Some of these sera also inhibited the growth of erythrocytic forms of $P$. falciparum in vitro (WILSON \& PhILlips, 1976). There have been a number of studies on the circulating malaria antigens recognized by the serum antibodies of individuals living in endemic areas (MCGREGOR et al., 1968; WILSON et al., 1975), but little is known about the antigens of $P$. falciparum erythrocytic forms which are recognized by these sera. The aim of this work is to identify the malaria antigens present in the various erythrocytic developmental stages of $P$. falciparum and which are recognized by the sera of individuals with varying degrees of immunity.

An isolate of $P$. falciparum prepared from parasitized blood of a European patient returning from Senegal (SGE 1) was adapted to in vitro culture according to the method of TRAGER \& JENSEN (1976). The culture was synchronized twice at 34-hour intervals by plasmagel sedimentation (REESE et al., 1979) and by incubation in 5\% manitol to lyse mature erythrocytic forms (LAMBROS \& VANDERBERG, 1979). The synchronized culture showed more than $98 \%$ homogeneity for the successive developmental stages of $P$. falciparum (ring forms, trophozoites and schizonts).

Synchronized cultures were sequentially pulselabelled for four hours with ${ }^{35} \mathrm{~S}$-methionine (specific activity: $239 \mathrm{TBq} / \mathrm{mmol} ; 20 \mu \mathrm{Ci} / \mathrm{ml}$ of culture medium; $4 \%$ suspension of human RBC with $9 \%$ parasitaemia). Labelled parasitized RBC were harvested, washed twice in phosphate saline buffer and lysed in seven volumes of $0.5 \%$ NonidetP40 in Tris NaC1-EDTA buffer. The lysate was centrifuged at $20,000 \mathrm{~g}$ for $20 \mathrm{~min}$ at $4^{\circ} \mathrm{C} . \quad 85$ to $90 \%$ of the label was present in the supernatant and 8 to $15 \%$ in the pellet. The supernatant of each stage-specific culture lysate was used as antigen source of the various $P$. falciparum erythrocytic stages. The total incorporation into TCA precipitable material was respectively $0.7 \%$, $4.6 \%$ and $6.6 \%$ in ring forms, trophozoites and schizonts and less than $0.1 \%$ in normal RBC.

*Supported by WHO, the UNAP/World Bank/WHO Special Program for Research and Training in

Tropical Diseases and the Swiss National Foundation (Grant No. 3.890.0.79). 
Supernatants of stage-specific culture lysates and of a non-synchronized culture lysate were analysed by SDS PAGE (LAEMMLY, 1970) followed by autoradiography (Fig. 1A). More than 40 labelled polypeptides can be identified, most of which are common to the different developmental stages of $P$. falciparum. However, some of the labelled bands probably represent stage-specific proteins. Three polypeptides of apparent molecular weights of 100,96 and $76 \mathrm{k}$-daltons are predominant in ring form preparations. Five polypeptides of apparent molecular weight of $100,96,76,68$ and $60 \mathrm{k}$-daltons are either predominant or specific for the trophozoite preparations. Polypeptides of $220,200,137,82$ and $45 \mathrm{k}$-daltons are either predominant in, or specific for, schizont preparations.

Supernatants of NP-40 lysates containing ${ }^{35}$ S-methionine-labelled $P$. falciparum proteins were used in double immunoprecipitation experiments (PerRIN et al., 1980). Two types of antisera were used as first antibody source: (a) 40 immune sera from Africans living in endemic areas for malaria (the Gambia and Tanzania); (b) 10 sera from Europeans recovering from a first attack of $P$. falciparum infection.

The second antibody was replaced by protein A which precipitates antibody-antigen complexes. Identical numbers of TCA precipitable counts $(250.000 \mathrm{cpm} / \mathrm{min}$.) of the NP-40 supernatants from the different developmental stages of $P$. falciparum were used as stage-specific antigen source and the optimal concentration of each serum containing anti- $P$. falciparum antibodies was determined in preliminary experiments. The washed immune precipitates were analysed by SDS PAGE followed by autoradiography. Fig. $1 B$ shows the results of one representative experiment, using three $P$. falciparum antigenic preparations labelled at various developmental stages and both types of human sera. The pattern of $P$. falciparum antigens precipitated by these two types of immune sera is representative of their respective groups.

It clearly appears that both types of sera, (a) from Africans and (b) from Europeans recovering from a
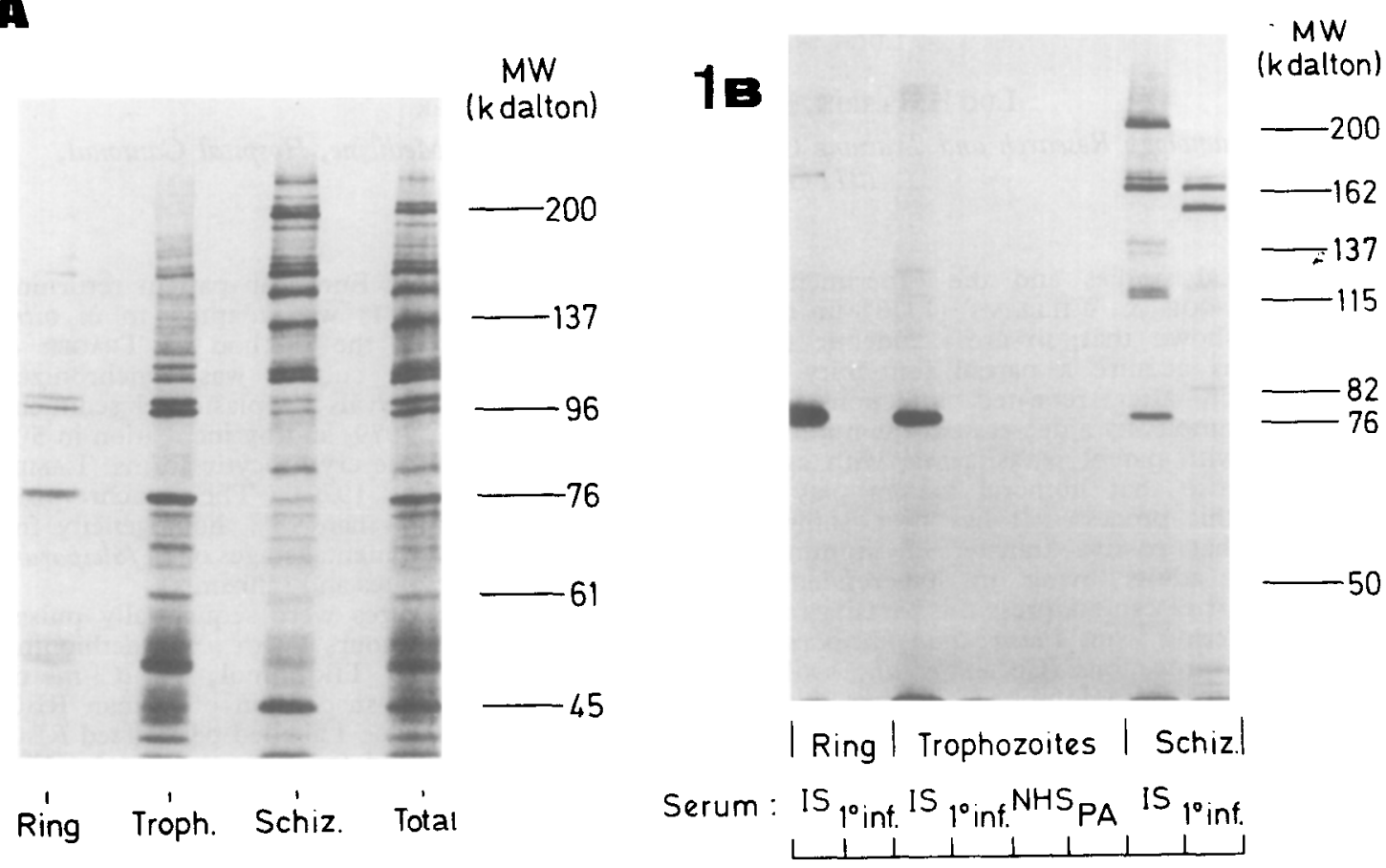

Fig. 1A. Autoradiography of SDS PAGE of NP-40 supernatants of the various developmental stages of $P$. falciparum and of a non-synchronized culture labelled with ${ }^{35} \mathrm{~S}$-methionine. Samples were diluted in starking gel buffer containing $2.5 \% 2$-mercaptoethanol and $1.5 \%$ SDS, boiled for $5 \mathrm{~min}$ at $80^{\circ} \mathrm{C}$ and analysed on $7.5 \%$ polyacrylamide slab gels. A $P$. falciparum culture synchronized by two manitol treastments was divided into three $45 \mathrm{~mm}$-petri dishes and used as the starting material for the ring, trophozoite and schizont preparations. The parasitaemia was $8.5 \%$ and a $5 \%$ suspension of $\mathrm{RBC}$ in $4 \mathrm{ml}$ was used for each labelling experiment. The cultures were pulse-labelled at various intervals after the second treatment with manitol : from 3 to 7 hours (ring forms), from 16 to 20 hours (trophozoites) and from 28 to 32 hours (schizonts). Parasitaemia was monitored with Giemsa-stained thin blood smears.

Fig. 1B. Autoradiography of immune precipitates analysed by $7.5 \%$ SDS slab PAGE. Two sera were used for the immunoprecipitation experiment : the serum from a European ( $1^{\circ}$ inf.) 10 days after recovery from a first $P$. falciparum infcction treatcd with chloroquine, and the serum from an adult African (IS) from Kenaba village (the Gambia) situated in an area hyperendemic for malaria. The titres of these two sera by indirect immunofluorescence on $P$. falciparum-parasitized RBC fixed by acetone were respectively 1:1024 and 1:4096. The sera were used as first antiserum against NP40 supernatants of various developmental stages of $P$. falciparum. Molecular weights are indicated in k-daltons on the left. 
first infection, precipitate mainly antigens from the schizont preparation, although the African immune sera precipitate the characteristic 76,000 dalton polypeptide from the ring and trophozoite preparations. Some polypeptides from the schizont preparation are selectively precipitated by the African immune serum. Their apparent molecular weights are 200, 137 (double bands), 115 (double bands) and $76 \mathrm{k}$-daltons. The polypeptides of molecular weights 200,137 and 115 are specific for the schizont stage. The 200 and $137 \mathrm{k}$-dalton polypeptides were selectively precipitated by most of the African immune sera analysed. Interestingly, the serum from the individual recovering from a first infection reacted with two polypeptides not recognized by the serum from the African immune sera, one of which had an apparent molecular weight of $82 \mathrm{k}$-daltons. This polypeptide was also precipitated by half of the sera from people living in areas endemic for malaria.

This report demonstrates that the incorporation of ${ }^{35} \mathrm{~S}$-methionine in $P$. falciparum polypeptides is maximum at the trophozoite and schizont developmental stages. Since mature crythrocytes do not synthesize proteins, all radioactive proteins from the labelled preparations are of parasite origin. Some polypeptides are characteristic or stage specific. Our results in this respect confirm and extend the earlier results of KILEJIAN (1979). We also detected a schizont-specific polypeptide of molecular weight $82 \mathrm{k}$-dalton which may be analogous to the $80 \mathrm{k}$-dalton polypeptides associated with knobs (KILEJIAN, 1979).

It is known that sera of adults living in areas endemic for malaria have precipitating antibodies against malarial antigens present in the serum of infected individuals and that there is a relationship between the presence of some of these antibodies and the degree of immunity (WILson et al., 1969, 1975; MCGREgor \& WILLIAMS, 1978). We found similarly that sera from adults living in endemic areas but not from individuals recovering from a first infection selectively recognized schizontspecific antigens. There is thus a qualitative difference in the antibody response toward antigens of the erythrocytic cycle of $P$. falciparm between adults living in endemic areas and adults recovering from a first infection. It is possible that the antigens specifically recognized by adults living in endemic areas are poorly immunogenic, and that repeated infections are needed for the induction of an antibody response against them.

Most of the polypeptides synthesized during the schizont stage of $P$. falcipartm are likely to be present in merozoites since schizonts release merozoites after rupture of the parasitized RBC.

Therc are two main limitations to this approach: (a) although supernatants used in the immunoprecipitation experiments contain more than $85^{\prime \prime}$ ', of the radiolabelled material, the possibility that some of the polypeptides are not solubilized cannot be excluded; (b) the localization of the immunoprecipitated antigens in the organism cannot be determined since our antigenic preparations contained both cytoplasmic and membrane-associated polypeptides of a given developmental stage. Evidently, polypeptides expressed on the surface of schizonts and merozoites are more likely to play a role in immune defence mechanisms of the host.
With the above limitations in mind, it can be postulated that the $P$. falciparum polypeptides recognized specifically in the schizont preparation (MW 200, 137, $115 \mathrm{k}$-daltons) by sera from adults living in endemic areas are present in the merozoites. Further work is also in progress to purify these polypeptides and to assess their possible role as antigens, which may be involved in protective immune responses toward the erythrocytic stages of P. falciparum.

\section{Acknowledgements}

The authors gratefully thank Dr. Ian McGregor (MRC Laboratory, The Gambia) and Dr. Lindsay Martinez-Mackey (WHO Research and Training Centre, Geneva) for providing sera from adults living in The Gambia.

\section{References}

Cohen, S., McGregor, I. A. \& Carrington, S. P. (I961). Gamma globulin and acquired immunity to human malaria. Nature, 192, 733-737.

Kilejian, A. (1979). Characterisation of a protein correlated with the production of knob-like protrusions on membrane of erythrocytes infected with Plasmodium falciparum. Proceedings of the National Academy of Sciences of the USA, 76, 4650-4653.

Laemmly, U.K. (1970). Clearage of structural proteins during the assembly of the head of bacteriophage T4. Nature, 227, 680-682.

Lambros, C. \& Vanderberg, J. P. (1979). Synchronisation of Plasmodium falciparum erythrocytic stages in culture. Journal of Parasitology, 65, 418-420.

McGregor, I. A. \& Williams, K. (1978). Value of the gel-precipitation test in monitoring the endemicity of malaria in a rural African village. Israel Fournal of Medical Sciences, 14, 697-706.

McGregor, I. A., Turner, M. W., Williams, K. \& Hall, P. (1968). Soluble antigens in the blood of African patients with severe Plasmodium falciparum malaria. Lancet, i, 881-884.

Perrin, L. H., Ramirez, E., Liu Er-Hsiang \& Lambert, P. H. (1980). Plasmodium falciparum: characterisation of defined antigens by monoclonal antibodies. Clinical and Experimental Immunology, (in press).

Reese, R. T., Langreth, S. G. \& Trager, W. (1979). Isolation of stages of the human parasite Plasmodium falciparum from culture and from animal blood. Bulletin of the World Health Organization, 57, (Suppl. No. 1), 53-61.

Trager, W. \& Jensen, J. B. (1976). Human malaria parasites in continuous culture. Science, 193, 673-675.

Wilson, R. J. M., McGrcgor, I. A., Hall, P. J., Williams, K. \& Bartholomew, R, (1969). Antigens associated with Plasmodium falciparum infection in man. Lancet, ii, 201.

Wilson, R. J. M., McGregor, I. A. \& Williams, K. (1975). Occurrence of S-antigens in serum in Plasmodium falciparum infections in man. Transactions of the Royal Society of Tropical Medicine and Hygiene, 69, 453-459.

Wilson, R. J. M. \& Phillips, R. S. (1976). Method to test inhibitory antibodies in human sera to wild populations of Plasmodium falciparum. Nature, 263, 132-134. 